\title{
ZUR ÜBERSETZUNG JURISTISCHER TERMINI IN DEUTSCHEN UND POLNISCHEN KLAGEANTRÄGEN
}

\author{
MICHAL MLODECKI \\ Adam Mickiewicz University - Poznañ
}

ABSTRACT. The present paper aimed at suggesting Polish and German equivalents of legal terms used in the petitions of suits in both languages. First the methods of translating legal terms given in the literature were presented. According to them came the catalogue of the mentioned Polish and German legal terms with the argumentation for the choice of the suggested equivalents both in Polish and German. The assumed principle was, that in the case of two different legal systems, one should, if possible, avoid translating legal terms of the source language with the parallel legal terms of the target language. The discussed legal terms and their equivalents were juxtaposed in a table as a summary.

Das Übersetzen im Fachbereich Recht ist ein immer aktuelles Thema, das noch bei weitem nicht erschöpft wurde. Besonders im Hinblick auf die wirtschaftliche Globalisierung und eine rasche Rechtsentwicklung auf der europäischen Ebene gewinnt das Übersetzen juristischer Fachtexte an Bedeutung. Diesem Zustand entspricht eine intensive Entwicklung der Rechtslinguistik, die zu einer Ebene der Zusammenarbeit zwischen der Juristen und der Sprachwissenschaftler wurde.

$\mathrm{Zu}$ betonen ist, daß mit dem Übersetzen der Texte in der Rechtssprache spezifische Schwierigkeiten verbunden sind, die beim Übersetzen anderer fachsprachlicher Texte nicht auftreten, bzw. in einem kleineren Ausmaß erscheinen. Das hat zu der unter Praktikern herrschenden Meinung zum hohen Schwierigkeitsgrad solcher Übersetzungen geführt, nach der

Legal translation, especially when we talk about the translation of legislation, can be extremely difficult task, as all of us doing it well know (Lehto 1988:431; zit. n. Hebenstreit 1997:97).

In diesem Aufsatz werden zwar keine legislativen Texte, sondern Texte aus dem Bereich der Rechtsanwendung untersucht, deren Übersetzung wirft jedoch analoge Probleme auf. 
Die meisten Schwierigkeiten bringt die Übersetzung von juristischen Termini mit sich. Man kann sagen, daß das Recht eine Ebene der sozialen Praxis ist, an die eine bestimmte Sprachverwendung gebunden ist. Neben dem Recht funktionieren andere Ebenen wie etwa Medizin, Sport, Politik oder Wirtschaft, die je über ihre eigenen Register verfügen. Im Unterschied zu ihnen ist die Ebene des Rechts nicht nur sprach-, sondern auch kulturspezifisch. Rechtssysteme entwickeln sich in jedem Land als Bestandteile seiner Geschichte und Kultur und werden den jeweils landesspezifischen Anforderungen der sozialen Wirklichkeit angepaßt. Die Tatsache, daß Rechtssysteme in der sozialen Wirklichkeit der Länder, in denen sie gelten, tief verankert sind, bedingt Inkongruenzen im Bereich der Terminologie des Rechts.

Im Verhältnis Sprache - Rechtssysteme sind folgende Konstellationen möglich: verschiedene Sprachen bei gleichem Rechtssystem, verschiedene Sprachen bei verschiedenen Rechtssystemen und gleiche Sprache bei verschiedenen Rechtssystemen. Mit dem ersten Fall hat man es in Staaten mit mehrere offiziellen Sprachen wie Kanada oder die Schweiz zu tun. Die Übersetzung bereitet dann keine besonderen Probleme, denn die Juristen operieren mit den gleichen Denotaten der Rechtstermini. Anders ist es im dritten Fall, in dem die Sprache zwar gleich ist, die Rechtssysteme aber unterschiedlich sind; zum Beispiel in verschiedenen deutschsprachigen oder englischsprachigen Ländern.

Sowohl im zweiten als auch im dritten Fall hat der Übersetzer zwischen zwei Welten zu vermitteln, wodurch er auch kulturellen Transfer vollzieht. Anders als bei den meisten modernen Fachsprachen, deren Terminologie weitgehend internationalisiert ist, stellt die erwähnte terminologische Inkongruenz die Hauptschwierigkeit des Übersetzens juristischer Texte dar.

Für die Übersetzung von Rechtstermini, die Namen von Rechtsinstituten sind, werden vier Verfahren eingesetzt:

- Übersetzung durch ein funktionales Äquivalent,

- die Verwendung des ursprünglichen oder transkribierten Terminus der Ausgangssprache mit einer eventuellen Erläuterung in Klammern oder in einer Fußnote mittels einer „wörtlichen Übersetzung“" oder einer Anmerkung wie „vgl. ...“,

- Umschreibung des ausgangssprachlichen Terminus in der Zielsprache,

- Wiedergabe durch einen Neologismus, d.h. durch einen Namen, der nicht zur zielsprachlichen Terminologie der Rechtsordnung gehört, eventuell in Kombination mit einer Erläuterung in einer Fußnote.

Die Komplexität der Rechtsinstitute, die immer eine jeweils spezifische Konstellation mehrerer rechtserheblicher Tatsachen bilden, führt zu der Ansicht, völlige Äquivalenz sei in Übersetzungen der die Rechtsinstitute bezeichnenden Termini nicht möglich. Nach de Groot/Schulze liegt nahezu völlige Äquivalenz vor, wenn: 
- von einer teilweisen Vereinheitlichung der für die Übersetzung relevanten Rechtsgebiete der einschlägigen Ausgangs- und Zielrechtsordnung die Rede sein kann;

- in der Vergangenheit ein Konzept aus der einen Rechtsordnung in die andere übernommen wurde und dort noch, unverändert und von dem übrigen Rechtssystem kaum beeinflußt, gilt. Eine Vielzahl von Beispielen läßt sich in Rechtsystemen finden, die das Recht eines anderes Rechtssystems rezipiert haben. Im Bereich des Privatrechts könnte man an die Türkei und die Schweiz denken.

Wenn Ausgangssprache und Zielsprache sich auf unterschiedliche Rechtssysteme beziehen, wie im Falle des Deutschen und des Polnischen, scheint (nahezu) völlige Äquivalenz jedoch problematisch zu sein.

Šarčević geht davon aus, daß es eine Übereinstimmung in wesentlichen Merkmalen zwischen zwei Rechtsbegriffen geben muß, damit der eine Terminus als funktionales Äquivalent des anderen gelten kann, und nimmt drei Kriterien an, nach denen man das Bestehen dieser Übereinstimmung prüfen kann. Diese Kriterien sind: ,scope of application, structure/classification und legal effect" (vgl. Šarčević 1989:108).

"The scope of application of a concept determines the cases to which the concept applies" (Šarčević 1989:283f.). Oft hat ein Rechtsinstitut in der Ausgangssprache einen größeren oder kleineren Anwendungsbereich als das analoge Rechtsinstitut in der Zielsprache. Dann ist ein Rechtsterminus als funktionales Äquivalent zu wählen, der ein Rechtsinstitut mit möglichst dem gleichen Anwendungsbereich denotiert.

Anhand der Kategorie structure/classification wird prüfbar, ob die funktionalen Äquivalente die gleichen Stellen in ihren Rechtssystemen einnehmen, weil die Rechtsinstitute im wesentlichen durch ihre Zugehörigkeit zu bestimmten Rechtsgebieten erkannt werden. Das gewählte funktionale Äquivalent sollte daher zum selben Rechtsbereich gehören wie der ausgangssprachliche Terminus.

Die letzte von Šrčević vorgeschlagene Kategorie legal effect bezieht sich auf die Rechtsfolgen, die die Anwendung der in Frage kommenden Rechtsinstitute in der Ausgangs- und in der Zielkultur herbeiführt. Diese Kategorie scheint mir bei der Entscheidung über die Wahl eines funktionalen Äquivalents die wichtigste zu sein, denn ein Fehler in dieser Hinsicht kann schwerwiegende Folgen haben. Man sollte daher vor allem die jeweiligen Umstände vor Augen haben. Das in einem Text zutreffende Äquivalent kann in einer anderen Situation unzutreffend sein. So ist beispielsweise die Übersetzung des Terminus Rechtsanwalt ins Polnische mit adwokat in vielen Fällen durchaus akzeptabel. Dieselbe Übersetzung in einem Schriftsatz aus einem deutsch-polnischen Rechtsstreit würde allerdings implizieren, daß es sich um einen Rechtsanwalt handelt, der zur Ausübung dieses Berufes in Polen berechtigt ist, was einer wesentlichen Änderung der Tatsachen gleichkäme. 
Eine Sammlung von Regeln für die Übersetzung von Rechtstexten hat der polnische Verband der Ökonomischen, Juristischen und Gerichtlichen Übersetzer und Dolmetscher veranlaßt (vgl. Kierzkowska 1991). Einer der Grundsätze dieser Regeln besagt, daß in der Übersetzung bestimmte sprachliche Konventionen der Zielfachsprache im Bereich der Phraseologie und der Terminologie nicht verletzt werden dürfen, unter der Annahme, daß der Empfänger ein Grundfachwissen auf dem gegebenen Rechtsgebiet besitzt. Für die Übersetzung juristischer Texte bedeutet das, daß als Empfänger ein Jurist gilt, der sich der Unterschiede zwischen zwei Rechtssystemen bewußt ist. Gleichzeitig sollte der Übersetzer den ständigen Bezug auf die Ausgangskultur aufrecherhalten. Auch in diesem Fall ist m.E. davon auszugehen, das der jeweilige Zweck der Übersetzung über den Kompromiß zwischen diesen zwei Grundsätzen entscheiden wird.

Das Beibehalten des ausgangssprachlichen Terminus ist dann zu empfehlen, wenn es eine besonders große Diskrepanz zwischen der Ausgangs- und der Zielrechtsordnung gibt, wie z.B. zwischen dem polnischen Rechtssystem und der Rechtsordnung der angelsächsischen Staaten, und wenn die ausgangssprachlichen Termini im Ausland bereits etabliert sind. Ein Beispiel eines solchen Falles ist der Terminus common law, der in polnischen Texten unübersetzt gelassen wird.

Bei Umschreibungen können wir von einem deskriptiven Äquivalent sprechen, wenn die gegebene Umschreibung in der Zielsprache eine nahezu perfekte Definition der zielsprachlichen Begriffes darstellt. Bei auftretenden Mängeln ähnelt dieses Verfahren der Verwendung eines Neologismus. In der Literatur wird zurecht die Meinung vertreten, die Zweckmäßigkeit der Umschreibung als Äquivalent hänge sowohl von der Länge und der Komplexität der Umschreibung als auch vom Ziel der Übersetzung ab.

Mit einem Neologismus haben wir es nach de Groot/Schulze dann zu tun, wenn in der Zielsprache einen Terminus benutzt wird, der in der einschlägigen Rechtsordnung der Zielsprache nicht (oder nicht mehr) verwendet wird. Ich schließe mich seiner Meinung an, nach der jeder Terminus, der nicht zur Terminologie der Zielrechtsordnung gehört, als Neologismus anzusehen ist. Als besonders geeignet für die Wahl eines Neologismus nennt er dabei Rechtstermini, die in der Ausgangsrechtsordnung früher eine äquivalente Bedeutung hatten oder aus der Terminologie anderer Rechtssysteme stammen, in denen die einschlägige Zielsprache als Rechtssprache benutzt wird. Die polnischen Rechtstermini könnten demgemäß mit entsprechenden Rechtstermini aus der österreichischen oder schweizerischen Rechtsordnung ins Deutsche übersetzt werden. Mit diesen Termini ist ein deutscher Jurist vertraut, zugleich wird ihm der notwendige Eindruck der Fremdheit vermittelt.

Im folgenden werden die signalisierten Aspekte der Übersetzung anhand von Rechtstermini aus deutschen und polnischen Klageanträgen erörtert. 
Klageanträge bilden einen Teil von Klageschriften, die zur Textsorte „Schriftsätze“ gehören. Man kann nach Busse sagen, daß Schriftsätze zu juristischen Textsorten i.e.S. gehören, für die folgende Merkmale kennzeichnend sind:

- Sie werden innerhalb der Institutionen des Rechtwesens und der Justiz von juristisch ausgebildeten und legitimierten Vertretern der Institutionen zu juristischen Zwecken als an juristische oder außerjuristische Adressaten gerichtete Texte produziert,

- Sie werden von nicht juristisch ausgebildeten Produzenten zu juristischen Zwecken als an institutionelle Adressaten gerichtete Texte produziert.

Für die Schriftsätze trifft auch das zweite Merkmal zu, da sie von $\mathrm{nicht}$ notwendig juristisch ausgebildeten Produzenten an den institutionellen Adressaten, d.h. das Gericht, gerichtet sind. Busse situiert anwaltliche Schriftsätze in der Kategorie der "Textsorten des Rechtfindungsverfahrens“. Die Rechtsterminologie läßt sich in folgende fünf Kategorien einteilen:

- die Terminologie der vom Gesetzgeber eingeführten juristischen Termini,

- die Terminologie der Rechtssprechung,

- die Terminologie der Bezeichnungen für Personen, die das Recht praktizieren,

- die Terminologie der rechtswissenschaftlichen Literatur,

- die Terminologie der Medien, sofern diese über das Rechtssystem bzw. Rechtsangelegenheiten berichten.

Entsprechend dieser Kategorisierung fällt die Terminologie der Schriftsätze unter die erste und die dritte Kategorie. Die Rechtstermini, deren Übersetzung ich erörtern möchte, sind dem Zivilprozeßrecht entnommen.

Für die in deutschen Klageschriften vorkommenden Fachbegriffe kann man in deren polnische Übersetzung folgende funktionale Äquivalente einsetzen:

$\begin{array}{ll}\text { Kläger } & - \text { powód } \\ \text { Beklagte } & - \text { pozwana } \\ \text { Klageschrift/Klage } & - \text { pozew/powództwo } \\ \text { Vollmacht } & - \text { pelnomocnictwo }\end{array}$

Für diese Beispiele sind analoge Benennung, definitorische Äquivalenz und analoge Rechtsfolgen kennzeichnend, d.h. zwischen ihnen besteht 1:1-Äquivalenz. Anzumerken ist allerdings der Unterschied im Gebrauch der detuschen Termini Klageschrift/Klage und der polnischen Termini pozew/powództwo. Der jeweils zweite Terminus dieser Paare bezeichnet die Handlung der Klageeinlegung, der jeweils erste dagegen das Dokument selbst. Während im Polnischen der entsprechende Schriftsatz konventionell die Überschrift Pozew trägt, benutzt 
man nach der deutschen Konvention das Wort Klage. Für die Verwendung der funktionalen Äquivalente spricht hier, daß diese Rechtsinstitute grundlegend für jedes System des Zivilprozeßrechts sind. Ihre Regelung ist im Vergleich zu anderen behandelten Rechtsinstituten knapp, weshalb es wenig wahrscheinlich ist, daß in dieser Hinsicht gravierende Unterschiede zwischen den einzelnen Zivilprozeßordnungen bestehen.

Im Falle der folgenden Begriffe lassen die Unterschiede m.E. die Verwendung funktionaler Äquivalente nicht zu, da sich die Gerichte der ersten und der zweiten Instanz von Staat zu Staat sowohl in Bezug auf das Sachenregister ihrer Zuständigkeit unterscheiden als auch hinsichtlich der Besetzung für die Rechtsstreite. Die innere Organisation der Gerichte regeln sowohl in Polen als auch in Deutschland besondere Gesetze, und zwar das Gerichtsverfassungsgesetz bzw. Prawo o ustroju sqqdów powszechnych. Somit ist sie für jeden Staat spezifisch. Auch die Prozeßnormen sind staatsspezifisch. Nach deutschem Recht ist in bestimmten Fällen beispielsweise die sog. Sprungrevision gegen das Urteil eines Landgerichts an den Bundesgerichtshof zulässig, im polnischen Recht dagegen gibt es kein analoges Rechtsmittel. Aus der unterschiedlichen Gerichtsorganisation ergeben sich auch Konsequenzen für die Übersetzung der Bezeichnungen der einzelnen Spruchkörper. Beispielsweise entscheidet in Polen in Zivilsachen in erster Instanz ein Richter, während in Deutschland die Zivilkammer mit drei Berufsrichtern besetzt ist. Funktionieren in Deutschland Kammern für Handelssachen ausschließlich an Landgerichten, entscheiden in Polen in Handelssachen auch Gerichte der ersten Instanz (sqdy rejonowe). Durch eine Übersetzung mit einem funktionalen Äquivalent würde der falsche Eindruck entstehen, die Spruchkörper in Polen und Deutschland sind gleich aufgebaut. Auch bei den Rechtsbegriffen vorläufige Vollstreckbarkeit - rygor natychmiastowej wykonalności ind die einschlägigen Regelungsbereiche sehr umfassend. In der deutschen Zivilprozeßordnung (ZPO) behandelt die Frage der Vollstreckbarkeit deren achtes Buch mit insgesamt 241 Paragraphen, im polnischen Kodeks Postępowania Cywilnego das zweite Buch des zweiten Teiles, mit insgesamt 423 Artikeln. Die Unterschiede betreffen grundlegende Fragen wie etwa den Katalog der Fälle, in denen diese Rechtsmittel ergriffen werden können.

Rechtsinstitute wie Versäumnisurteil, Anerkenntnisurteil oder das schriftliche Vorverfahren sind im deutschen Recht anders geregelt als im polnischen, wobei sich die Unterschiede auf die Rechtsfolgen beziehen. Ein Versäumnisurteil wird nach deutschem Recht immer auf Antrag erlassen, wobei es sowohl gegen den Beklagten als auch gegen den Kläger ergehen kann. Nach polnischem Recht kann ein entsprechendes Urteil (wyrok zaoczny) nur gegen den Beklagten ergehen, auch ohne Antrag des Klägers. Um diesem Unterschied Rechnung zu tragen, erachte ich als Übersetzungsäquivalente Kadumazurteil bzw. Abwesenheitsurteil und wyrok w trybie zaocznym. 
Der Terminus Anerkenntnisurteil hat im polnischen Recht weder eine terminologisch noch eine begriffliche Entsprechung, obwohl das Rechtsinstitut mit analoger Funktion im Rechtssystem existiert. Man spricht dann ( $a d$ hoc) über ein im Fall des Anerkenntnisses der Klage zu erlassendes Urteil. Es kommt auch der Ausdruck wyrok z uznania (Urteil aufgrund der Anerkenntnis) vor, der als Terminus gelten könnte, aber nicht allgemein verwendet wird. Darüber hinaus gibt es Unterschiede im Bereich der Rechtsfolgen, denn das deutsche Recht bindet dieses Urteil an einen Antrag des Klägers, während das polnische Gesetz besagt, daß das Gericht durch Anerkenntnis gebunden ist, ohne daß dazu ein Antrag gestellt werden muß.

Die loseste Entsprechung aus dieser Gruppe der Rechtstermini kann man im polnischen Recht für den Terminus das schriftliche Vorverfahren finden. In der deutschen ZPO stellt dieses Rechtsinstitut eine von zwei Möglichkeiten dar, die der Richter nutzt, um die Hauptverhandlung vorzubereiten. Im Wege des schriftlichen Vorverfahrens fordert der Richter den Beklagten mit der Klagezustellung auf, die Verteidigungsabsicht binnen einer Notfrist von zwei Wochen nach Zustellung der Klageschrift dem Gericht schriftlich anzuzeigen. Dem Beklagte werden zugleich weitere zwei Wochen zur schriftlichen Klageerwiderung eingeräumt, auf die dann der Kläger eine schriftliche Stellungnahme formuliert. Im polnischen Recht gilt die Regel, daß der Beklagte die Klageerwiderung entweder vor oder in der ersten Verhandlung unterbreiten kann. Angeordnet werden kann die Abfassung der Klageerwiderung oder der Schriftenaustausch vor der ersten Verhandlung nur in komplizierten Rechtsstreiten.

So wie im Fall des Anerkenntnisurteils gibt es in der polnischen Rechtssprache keinen etablierten Terminus für diese Prozeßhandlung, hier rührt dies aber daher, daß dieser Schriftenaustausch gerade nicht als Rechtsinstitut betrachtet wird. Man könnte also zu einer Lehnübersetzung pisemne postepowanie wstepne oder zu einer beschreibenden Übersetzung wymiana pism (Schriftenaustausch) greifen. Die zweite vermittelt m.E. den Sinn des deutschen Rechtsinstituts besser.

Das letzte der angeführten Rechtsinstitute - der frühe erste Termin zur mündlichen Verhandlung - ist dem polnischen Recht unbekannt. Es gibt auch kein Verfahren mit ähnlicher Funktion. Im deutschen Recht ist es neben dem schriftlichen Vorverfahren gerade die zweite Möglichkeit für den Richter, den Haupttermin vorzubereiten. Sie besteht darin, daß beide Parteien vor Gericht geladen werden, um strittige Fragen mit dem Richter zu diskutieren und sich so möglichst effektiv auf den Haupttermin vorzubereiten. Um diesen Terminus in der polnischen Sprache wiederzugeben, ist eine Lehnübersetzung eigentlich ausgeschlossen, denn der an den Terminus gebundene Begriff ist für eine solche zu komplex. Es bleibt die umschreibende Übersetzung. Eine Möglichkeit wäre, den Begriff als przedterminowe postępowanie ustne (vorfristiges mündliches Verfahren) zu übersetzen. 
Nachstehende Tabelle stellt die besprochenen Vorschläge für die Übersetzung der deutschen und polnischen Rechtstermini dar:

\begin{tabular}{|c|c|c|c|}
\hline $\begin{array}{l}\text { Deutscher } \\
\text { Terminus }\end{array}$ & $\begin{array}{c}\text { Polnische } \\
\text { Entsprechung }\end{array}$ & $\begin{array}{l}\text { Polnischer } \\
\text { Terminus }\end{array}$ & $\begin{array}{l}\text { Deutsche } \\
\text { Entsprechung }\end{array}$ \\
\hline Klage/Klageschrift & powództwo/pozew & powództwo/pozew & Klage/Klageschrift \\
\hline Kläger/Beklagter & powód/pozwany & powód/pozwany & Kläger/Beklagter \\
\hline Vollmacht & petnomocnictwo & petnomocnictwo & Vollmacht \\
\hline Amtsgericht & sq̨d niższy/l instancji & sad rejonowy & Rayongericht \\
\hline Landgericht & sqd wyższy & sqd okregowy & Bezirksgericht \\
\hline Zivilkammer & wydziat cywilny & wydziat cywilny & Zivilabteilung \\
\hline $\begin{array}{l}\text { Kammer } \\
\text { für Handelssachen }\end{array}$ & wydziat handlowy & wydziat gospodarczy & Handelsabteilung \\
\hline $\begin{array}{l}\text { vorläufige Voll- } \\
\text { streckbarkeit }\end{array}$ & $\begin{array}{l}\text { rygor tymczasowej } \\
\text { wykonalnosici }\end{array}$ & $\begin{array}{l}\text { rygor natychmiastowej } \\
\text { wykonalności }\end{array}$ & $\begin{array}{l}\text { sofortige Voll- } \\
\text { streckbarkeit }\end{array}$ \\
\hline Versäumnisurteil & $\begin{array}{l}\text { wyrok } w \text { trybie } \\
\text { zaocznym }\end{array}$ & wyrok zaoczny & Abwesenheitsurteil \\
\hline Anerkenntnisurteil & wyrok $z$ uznania & & \\
\hline $\begin{array}{l}\text { das schriftliche } \\
\text { Vorverfahren }\end{array}$ & $\begin{array}{l}\text { pisemne postepowanie } \\
\text { przygotowawcze }\end{array}$ & & \\
\hline $\begin{array}{l}\text { der frühe erste Ter- } \\
\text { min zur mündlichen } \\
\text { Verhandlung }\end{array}$ & $\begin{array}{l}\text { wstepne postępowanie } \\
\text { ustne }\end{array}$ & & \\
\hline
\end{tabular}

Das Gesagte macht sichtbar, daß das Übersetzen von Texten der deutschen Rechtssprache ins Polnische trotz vieler Übereinstimmungen zwischen beiden Rechtssystemen einige zumindest allgemeine Rechtskenntnisse erfordert, denn nur unter dieser Voraussetzung kann der Übersetzer optimale Entscheidungen darüber treffen, welches der zur Verfügung stehenden Übersetzungsverfahren er anzuwenden hat. Erforderlich ist allerdings auch die Einarbeitung in die beiden Rechtssysteme und der Vollzug vergleichender Begriffsanalysen, um in diesem Bereich auf jeden Fall Sicherheit zu gewinnen. Es gäbe sonst auch keine festen Kriterien für die Prüfungen, die zur Zeit Kandidaten für die Lizenz eines Gerichtsdolmetschers bzw. -übersetzers in Warschau und Poznań abzulegen haben. Es besteht daher Bedarf nach einer intensiven Zusammenarbeit von Juristen (Richter, Rechtswissenschaftler) und Sprachwissenschaftlern. Hilfreich wäre auch die internationale Standardisierung von Übersetzungsäquivalenten, zumin- 
dest für die zentralen Rechtsbegriffe der einzelnen nationalen Rechtssysteme. Neben der Terminologiearbeit sollten kontrastive Textsortenanalysen, die u.a. die Gestalt und die Funktion von Texten im juristischen Bereich untersuchen, fruchtbare Effekte liefern.

\section{LITERATUR}

Busse, Dietrich (2000): Textsorten des Bereichs Rechtswesen und Justiz. In: Klaus Binker et al.

(Hg.), Text- und Gesprächslinguistik. Ein internationales Handbuch zeitgenössischer Forschung. 1 Halbbd. Berlin - New York: de Gruyter, 658-675.

Encyklopedia prawa (1999). Warszawa.

de Groot, Gerard/Schulze, Reiner (1999): Recht und Übersetzen. Baden-Baden.

Hebenstreit, Gernot (1997): Terminus - Weltbild - Intertextualität: Translatorische Überlegungen zu juristischen Fachtexten. In: N. Grbić/M. Wolf (Hg.), Text - Kultur - Kommunikation. Translation als Forschungsaufgabe. Tübingen.

Kierzkowska, Danuta (1991): Kodeks ttumacza sadowego. Warszawa.

Lehto, Leena (1998): The Need for Standardisation of Concept Equivalents in The Field of law. In: P. Nekeman (Hg.), 431-437.

Stein, Stephan (1995): Formelhafte Sprache: Untersuchungen zu ihren paradigmatischen und kognitiven Funktionen im gegenwärtigen Deutsch. Frankfurt a.M. 